\title{
Prolific authors and institutions in leading international business journals
}

\author{
Shichun Xu • Goksel Yalcinkaya • Steven H. Seggie
}

Published online: 17 November 2007

(C) Springer Science + Business Media, LLC 2007

\begin{abstract}
The science and practice of international business is advanced through scholarly contribution to the leading international business journals. Increased competition among academic institutions has led to increased emphasis on publication in the leading international business journals. Yet, little is known as to the answers to questions such as: (1) Who are the most prolific authors in the leading international business journals? and (2) Which educational institutions appear poised to lead international business scholarship? To examine these questions, the study examines scholarly work in international business over the past eleven years in six leading international business journals (i.e., Journal of International Business Studies, Management International Review, Journal of World Business, International Marketing Review, Journal of International Marketing, and International Business Review). Addressing these two research questions provides us with a more complete understanding of the authors and institutions most likely to influence the field of international business.
\end{abstract}

Keywords International business scholarship · Prolific authors · Leading schools

As the international business discipline garners greater prominence in the academic community, it is now subject to the same quantification as other disciplines. Evidence of

The authors are grateful to S. Tamer Cavusgil and David A. Griffith for their significant contribution during the research and Mike W. Peng (Editor-in-Chief) for his detailed editorial assistance.

S. $\mathrm{Xu}$

Department of Marketing and Logistics, College of Business Administration,

University of Tennessee, 310 Stokely Management Center, Knoxville, TN 37996, USA

e-mail: sxu3@utk.edu

G. Yalcinkaya $(\bowtie)$

University of New Hampshire, Whittemore School of Business and Economics,

McConnell Hall, 15 College Road, Durham, NH 03824-3593, USA

e-mail: goksel.yalcinkaya@unh.edu

S. H. Seggie

Department of Management, Bilkent University, Ankara, Turkey

e-mail: steven@bilkent.edu.tr 
this includes institutional rankings based on research such as the University of Texas at Dallas School of Management rankings (http://citm.utdallas.edu.utdrankings/) or those provided by Trieschmann, Dennis, Northcraft, and Niemi (2000) allowing for the ranking of institutions based on research in international business journals. As these rankings are driven by publications by individual scholars within institutions, this increased emphasis on rankings necessitates an increased focus on the individuals publishing in the top international business journals. Furthermore, the publication records of individuals are heavily weighted when making promotion or tenure decisions. Academics decide what the realistic expectations are for an individual to be promoted to associate professor or full professor, yet little or no objective data have been offered to tell us whether or not these expectations are realistic. This lack of objective data ensures that as a field we are unable to discern what level of productivity is necessary to be deemed truly outstanding in comparison to one's peers.

This study serves as a useful extension of the previous studies that discussed the most prolific and cited authors in international business (Chan, Fung, \& Lai, 2005; Kumar \& Kundu, 2004; Meyer \& Peng, 2005; Peng, 2001; Peng \& Zhou, 2006; Pleggenkuhle, Aroul, Sun, \& Su, 2007). In addition, this paper follows the Griffith, Cavusgil, and $\mathrm{Xu}$ (2008) study that attempts to understand the drivers of international business research agenda as well as emerging themes that are likely to set the stage for future work in the literature. This study is driven by two research questions. First, who are the most prolific authors in the leading international business journals? Second, which educational institutions appear poised to lead international business scholarship? To address these questions, we examined author productivity within the six leading international business journals (Journal of International Business Studies, Management International Review, Journal of World Business, International Marketing Review, Journal of International Marketing, and International Business Review) from 1996 to 2006. Further, given the nature of publication and the importance of current affiliation we examined institutional influence based upon authors' current affiliation.

\section{Methodology}

Leading international business journals

Dubois and Reeb (2000) identified six journals as the leading journals in the field of international business (Journal of International Business Studies, Management International Review, Journal of World Business, International Marketing Review, Journal of International Marketing and International Business Review). Clear distinctions exist among these journals in terms of the number of articles published, number of article citations, number of issues per volume, and so on. Table 1 provides an overview of these journals.

Authorship analysis and institution identification

To address the two research questions posed in this study, the tables of contents of each of the six journals were used to develop a dataset. The objective was to focus 
Table 1 Overview of journals and articles (1996-2006).

\begin{tabular}{llllllll}
\hline & JIBS & MIR & JWB & JIM & IMR & IBR & Total \\
\hline Total articles published & 411 & 344 & 291 & 221 & 346 & 382 & 1,995 \\
Citations $^{\text {a }}$ & 4,873 & 881 & 1,104 & 933 & 745 & 62 & 8,598 \\
Average number of citations per article & 11.86 & 2.56 & 3.79 & 4.22 & 2.15 & 0.16 & 4.31 \\
Number of published scholars & 740 & 540 & 491 & 386 & 546 & 616 & 2,495 \\
Single author & 88 & 107 & 84 & 49 & 103 & 104 & 535 \\
Two authors & 182 & 155 & 117 & 94 & 144 & 169 & 861 \\
Three authors & 108 & 68 & 66 & 53 & 73 & 85 & 453 \\
Four authors & 21 & 13 & 18 & 18 & 17 & 22 & 109 \\
Five or more authors & 10 & 1 & 5 & 7 & 9 & 2 & 34 \\
Total number of separate issues & 53 & 66 & 44 & 44 & 61 & 64 & 332 \\
Average number of articles per issue & 7.75 & 5.21 & 6.59 & 5.02 & 5.67 & 5.88 & 6.02 \\
Average number of authors per article & 2.43 & 2.00 & 2.13 & 2.29 & 2.11 & 2.08 & 2.17 \\
\hline
\end{tabular}

${ }^{\text {a }}$ Based on Social Science Citation Index (SSCI) as of January 3, 2007

on articles that addressed international business research through quantitative, qualitative or theory papers. While editorials, commentaries and replies make legitimate contributions to the field of international business, they are often not reviewed under the same competitive review process as regular articles and, therefore, were excluded. Similar to Inkpen and Beamish (1994), editorials (including letters to the editor), special issue guest editorials, comments and replies were excluded. In addition, decisions were made pertaining to unique types of articles published in each journal. ${ }^{1}$

While analyzing the remaining articles two aspects were coded. First, each article was coded to identify the authors, thus providing an author count (appearance). Second, following Inkpen and Beamish (1994), an "adjusted authorship count" score was tabulated. Adjusted authorship count addresses multiple-author contributions. For example, whereas an author receives a score of " 1 " for a single-authored work, in an article published with two authors, each author received a score of " 0.5 "; in an article with three authors, each author received a score of " 0.333 "; and so on. One unique article within the dataset was in JIBS 33.4 (2002). An article in this issue listed four authors and a "network of others." In this case, authorship was coded as 5 authors with adjusted authorship being " 0.20 " per author. Results for each journal, as well as a cumulative total across the six leading international business journals, are reported. Following Inkpen and Beamish (1994), adjusted author count was used as the primary sort criterion (given the importance of author appearances in terms of prolific authorship, raw author counts are also provided).

\footnotetext{
${ }^{1}$ For example, JIBS Symposium introductions were treated as guest editorials and were excluded. JIBS Decade Award re-printed articles, as well as invited replies, were excluded. In JIBS 37.3 (2006), both the preface to the essay and the essay itself were excluded. We also excluded the conference report in JIBS 37.2 (2006). In Management International Review, the Executives Forum in issue 36.2 (1996) and Comments in 36.3 (1996) and 36.4 (1996) were excluded. In International Marketing Review, the Internet Forum comments (published in issues 18.4 (2001), 18.5 (2001), 18.6 (2001), 19.2/3 (2002) and 19.4/5 (2002)) were excluded. Invited Reflections on Czinkota and Ronkainen's International Marketing Manifesto in Journal of International Marketing 11.1 (2003) were excluded. Commentaries in International Business Review were excluded.
} 
To address the second research question, (i.e., what institutions are poised to drive the future direction of international business), information pertaining to an author's current institutional affiliation was obtained. In delineating institutional affiliation, one could examine the institution hosting an author at the time of article publication or, alternatively, the current institution hosting the author. As the purpose of this research is to not only identify those scholars driving the field of international business in the past, but also to identify those institutions likely to have the greatest influence in driving the future research agenda of international business, it was determined that the current institutional affiliation would be most appropriate for this study. Hence, the institutional affiliation of authors ranking in the top group of cumulative authorship (i.e., adjusted authorship scores of equal to or greater than 2.50) were identified using the Academy of International Business (AIB) membership directory (as the AIB membership directory is believed to provide the most up-to-date affiliations). Those individuals not appearing in the AIB membership directory were found through the Internet to identify their current institutional affiliation.

\section{Results}

Prolific authors in international business

The first research question asked who were the most prolific scholars in the leading international business journals. Tables 2, 3, 4, 5, 6 and 7 identify the most prolific authors in each of the six journals (based upon adjusted count greater than 1.00). Table 2 presents the results for the Journal of International Business Studies. Overall, 740 different authors appeared in JIBS over the 11-year time period (average 1.37 appearances, average 0.58 adjusted appearances). Of the 740 authors, 587 authors (79.3\%) appeared only once, 100 authors (13.5\%) appeared twice and 53 authors $(7.2 \%)$ appeared three times or more.

Table 3 presents the results for the Management International Review. Overall, 541 different authors appeared in MIR over the 11-year time period (average 1.26 appearances, average 0.63 adjusted appearances). Of the 541 authors, 451 authors $(83.3 \%)$ appeared only once, 60 authors (11.1\%) appeared twice and 30 authors $(5.6 \%)$ appeared three times or more.

Table 4 presents the results for the Journal of World Business. Overall, 491 different authors appeared in $J W B$ over the 11-year time period (average 1.25 appearances, average 0.59 adjusted appearances). Of the 491 authors, 415 authors $(84.5 \%)$ appeared only once, 46 authors $(9.4 \%)$ appeared twice and 30 authors $(6.1 \%)$ appeared three times or more.

Table 5 presents the results for the International Marketing Review. Overall, 552 different authors appeared in IMR over the 11-year time period (average 1.28 appearances, average 0.59 adjusted appearances). Of the 552 authors, 439 authors $(79.5 \%)$ appeared only once, 82 authors (14.8\%) appeared twice and 31 authors $(5.6 \%)$ appeared three times or more.

Table 6 presents the results for the Journal of International Marketing. Overall, 387 different authors appeared in JIM over the 11-year time period (average 1.31 
Table 2 Most prolific authors in Journal of International Business Studies (1996-2006): by adjusted count.

\begin{tabular}{|c|c|c|c|}
\hline Rank & Author & Count & Adjusted \\
\hline 1 & Luo, Yadong & 12 & 8.42 \\
\hline 2 & Buckley, Peter J. & 9 & 4.83 \\
\hline 3 & Pan, Yigang & 8 & 4.50 \\
\hline 4 & Brouthers, Lance Eliot & 8 & 3.50 \\
\hline 4 & Tse, David & 9 & 3.50 \\
\hline 6 & Shenkar, Oded & 6 & 3.42 \\
\hline 7 & Dunning, John H. & 3 & 3.00 \\
\hline 7 & Meyer, Klaus & 4 & 3.00 \\
\hline 7 & Peng, Mike W. & 5 & 3.00 \\
\hline 10 & Brouthers, Keith D. & 5 & 2.67 \\
\hline 10 & Kwok, Chuck C. & 6 & 2.67 \\
\hline 12 & Reeb, David M. & 5 & 2.33 \\
\hline 12 & Verbeke, Alain & 5 & 2.33 \\
\hline 12 & Kotabe, Masaaki & 6 & 2.33 \\
\hline 15 & $\mathrm{Au}, \mathrm{Kevin}$ & 4 & 2.17 \\
\hline 15 & Birkinshaw, Julian & 4 & 2.17 \\
\hline 15 & Makino, Shige & 5 & 2.17 \\
\hline 18 & Casson, Mark & 4 & 2.00 \\
\hline 18 & Ramamurti, Ravi & 2 & 2.00 \\
\hline 18 & Rangan, Subramanian & 2 & 2.00 \\
\hline 18 & Rugman, Alan M. & 4 & 2.00 \\
\hline 18 & Simonin, Bernard L. & 2 & 2.00 \\
\hline 18 & Spencer, Jennifer W. & 2 & 2.00 \\
\hline 18 & Sullivan, Daniel P. & 2 & 2.00 \\
\hline 25 & Lenartowicz, Tomasz & 4 & 1.83 \\
\hline 25 & Mudambi, Ram & 3 & 1.83 \\
\hline 25 & Newburry, William & 3 & 1.83 \\
\hline 25 & Pantzalis, Christos & 3 & 1.83 \\
\hline 25 & Reuer, Jeffrey J. & 3 & 1.83 \\
\hline 25 & Aulakh, Preet S. & 5 & 1.83 \\
\hline 25 & Werner, Steve & 5 & 1.83 \\
\hline 32 & Husted, Bryan W. & 3 & 1.75 \\
\hline 33 & Griffith, David A. & 4 & 1.67 \\
\hline 33 & Erramilli, M. Krishna & 3 & 1.67 \\
\hline 35 & Caves, Richard E. & 2 & 1.50 \\
\hline 35 & Chen, Shih-Fen S. & 2 & 1.50 \\
\hline 35 & Coviello, Nicole E. & 2 & 1.50 \\
\hline 35 & Delios, Andrew & 3 & 1.50 \\
\hline 35 & Dow, Douglas & 2 & 1.50 \\
\hline 35 & Ellis, Paul & 2 & 1.50 \\
\hline 35 & Feinberg, Susan E. & 2 & 1.50 \\
\hline 35 & Grosse, Robert & 2 & 1.50 \\
\hline 35 & Harvey, Michael G. & 2 & 1.50 \\
\hline 35 & Henisz, Witold J & 2 & 1.50 \\
\hline 35 & Hennart, Jean-Francois & 3 & 1.50 \\
\hline 35 & Inkpen, Andrew C. & 2 & 1.50 \\
\hline 35 & Money, R. Bruce & 2 & 1.50 \\
\hline 35 & Myers, Matthew B. & 2 & 1.50 \\
\hline 35 & Nachum, Lilach & 2 & 1.50 \\
\hline 35 & Nigh, Douglas & 3 & 1.50 \\
\hline 35 & Parkhe, Arvind & 3 & 1.50 \\
\hline 35 & Safarian, A Edward & 2 & 1.50 \\
\hline 35 & Uhlenbruck, Klaus & 2 & 1.50 \\
\hline 35 & Van de Vliert, Evert & 2 & 1.50 \\
\hline
\end{tabular}


Table 2 (continued)

\begin{tabular}{llll}
\hline Rank & Author & Count & Adjusted \\
\hline 35 & Yan, Aimin & 2 & 1.50 \\
35 & Cavusgil, S. Tamer & 4 & 1.50 \\
57 & Chung, Wilbur & 2 & 1.33 \\
57 & Globerman, Steven & 3 & 1.33 \\
57 & Hagedoorn, John & 3 & 1.33 \\
57 & Kundu, Sumit K. & 3 & 1.33 \\
57 & Roth, Kendall & 3 & 1.33 \\
57 & Scandura, Terri A. & 3 & 1.33 \\
57 & Vaaler, Paul M. & 3 & 1.33 \\
64 & Ralston, David A. & 4 & 1.28 \\
65 & Balabanis, George & 2 & 1.25 \\
65 & Diamantopoulos, Adamantios & 4 & 1.25 \\
67 & Ghemawat, Pankaj & 2 & 1.20 \\
67 & Hofstede, Geert & 2 & 1.20 \\
67 & Khanna, Tarun & 3 & 1.20 \\
70 & Lau, Chung-Ming & 3 & 1.17 \\
71 & Lyles, Marjorie A. & 3 & 1.08 \\
72 & Bjorkman, Ingmar & 3 & 1.03 \\
72 & Park, Seung Ho & 3 & 1.03 \\
\hline
\end{tabular}

Listing only includes authors with an adjusted count greater than 1.0. Overall, 740 different authors appeared in Journal of International Business Studies over the 11-year time period (average 1.37 appearances, average 0.58 adjusted appearances). Of the 740 authors, 587 authors $(79.3 \%)$ appeared only once, 100 authors (13.5\%) appeared twice and 53 authors (7.2\%) appeared three times or more.

appearances, average 0.57 adjusted appearances). Of the 387 authors, 318 authors $(82.1 \%)$ appeared only once, 46 authors $(11.9 \%)$ appeared twice and 23 authors $(6.0 \%)$ appeared three times or more.

Table 7 presents the results for International Business Review. Overall, 616 different authors appeared in IBR over the 11-year time period (average 1.29 appearances, average 0.62 adjusted appearances). Of the 616 authors, 504 authors $(81.8 \%)$ appeared only once, 77 authors (12.5\%) appeared twice and 35 authors $(5.7 \%)$ appeared three times or more.

Table 8 presents the cumulative results of authorship across the six international business journals and the affiliated institutions of the most prolific scholars (based upon adjusted count greater than or equal to 2.50). Overall, 2,495 different authors appeared in the six leading international business journals over the 11-year time period (average 1.73 appearances, average 0.80 adjusted appearances). Of the 2,495 authors, 1,755 (70.3\%) appeared only once, $372(14.9 \%)$ appeared twice, 368 (14.8\%) appeared between three times or more. For the purposes of APJM, authors affiliated with Asia Pacific schools are shown in bold.

\section{Leading educational institutions}

The second research question asked which educational institutions appeared poised to lead international business scholarship. Table 9 provides the current institutional affiliations of the most prolific authors in the six leading international business 
Table 3 Most prolific authors in Management International Review (1996-2006): by adjusted count.

\begin{tabular}{|c|c|c|c|}
\hline Rank & Author & Count & Adjusted \\
\hline 1 & Luo, Yadong & 6 & 4.50 \\
\hline 2 & Buckley, Peter J. & 8 & 4.42 \\
\hline 3 & Merchant, Hemant & 3 & 3.00 \\
\hline 3 & Tsang, Eric W. K. & 3 & 3.00 \\
\hline 5 & Mudambi, Ram & 5 & 2.25 \\
\hline 5 & Beamish, Paul W. & 6 & 2.25 \\
\hline 7 & Casson, Mark & 2 & 2.00 \\
\hline 7 & Enright, Michael J. & 2 & 2.00 \\
\hline 7 & Nachum, Lilach & 2 & 2.00 \\
\hline 7 & Rugman, Alan M. & 4 & 2.00 \\
\hline 7 & Selmer, Jan & 2 & 2.00 \\
\hline 7 & Witt, Peter & 2 & 2.00 \\
\hline 13 & Birkinshaw, Julian & 4 & 1.83 \\
\hline 13 & Dowling, Peter J. & 3 & 1.83 \\
\hline 13 & Sambharya, Rakesh B. & 4 & 1.83 \\
\hline 16 & Welch, Denice E. & 3 & 1.67 \\
\hline 16 & Welch, Lawrence S. & 4 & 1.67 \\
\hline 18 & Cavusgil, S. Tamer & 3 & 1.50 \\
\hline 18 & Glaister, Keith W. & 3 & 1.50 \\
\hline 18 & Grosse, Robert & 2 & 1.50 \\
\hline 18 & Moore, Karl J. & 2 & 1.50 \\
\hline 18 & Peng, Mike W. & 2 & 1.50 \\
\hline 18 & Rhee, Jay Hyuk & 2 & 1.50 \\
\hline 18 & Verbeke, Alain & 3 & 1.50 \\
\hline 25 & Choi, Chong Ju & 4 & 1.42 \\
\hline 26 & Abramson, Neil R. & 3 & 1.33 \\
\hline 26 & Brewster, Chris & 2 & 1.33 \\
\hline 26 & Cantwell, John & 3 & 1.33 \\
\hline 26 & Delios, Andrew & 3 & 1.33 \\
\hline 26 & Johanson, Jan & 3 & 1.33 \\
\hline 26 & Katsikeas, Constantine S. & 2 & 1.33 \\
\hline 26 & Leonidou, Leonidas C. & 2 & 1.33 \\
\hline 26 & Li, Lei & 2 & 1.33 \\
\hline 26 & Mahnke, Volker & 3 & 1.33 \\
\hline 26 & Pak, Yong Suhk & 3 & 1.33 \\
\hline 26 & Park, Young-Ryeol & 3 & 1.33 \\
\hline 26 & Robertson, Christopher J. & 2 & 1.33 \\
\hline 26 & Tallman, Stephen & 2 & 1.33 \\
\hline 26 & Venzin, Markus & 3 & 1.33 \\
\hline 26 & Wolf, Joachim & 2 & 1.33 \\
\hline 26 & Zhao, Hongxin & 3 & 1.33 \\
\hline 42 & Lin, Xiaohua & 2 & 1.25 \\
\hline 42 & Qian, Gongming & 2 & 1.25 \\
\hline 42 & Clegg, Jeremy & 4 & 1.25 \\
\hline 45 & De Cieri, Helen & 3 & 1.17 \\
\hline 45 & Pedersen, Torben & 3 & 1.17 \\
\hline 45 & Petersen, Bent & 3 & 1.17 \\
\hline
\end{tabular}

Listing only includes authors with an adjusted count greater than 1.00. Overall, 541 different authors appeared in Management International Review over the 11-year time period (average 1.26 appearances, average 0.63 adjusted appearances). Of the 541 authors, 451 authors (83.3\%) appeared only once, 60 authors $(11.1 \%)$ appeared twice and 30 authors (5.6\%) appeared three times or more. 
Table 4 Most prolific authors in Journal of World Business (1996-2006): by adjusted count.

\begin{tabular}{|c|c|c|c|}
\hline Rank & Author & Count & Adjusted \\
\hline 1 & Harvey, Michael G. & 8 & 3.92 \\
\hline 2 & Luo, Yadong & 3 & 3.00 \\
\hline 3 & Griffith, David A. & 5 & 2.58 \\
\hline 4 & Czinkota, Michael R. & 4 & 2.50 \\
\hline 5 & Janssens, Maddy & 4 & 2.17 \\
\hline 5 & McCarthy, Daniel & 5 & 2.17 \\
\hline 5 & Puffer, Sheila M. & 5 & 2.17 \\
\hline 8 & Cavusgil, S. Tamer & 4 & 2.08 \\
\hline 9 & Chang, Eunmi & 2 & 2.00 \\
\hline 9 & Parkhe, Arvind & 2 & 2.00 \\
\hline 9 & Vandermerwe, Sandra & 2 & 2.00 \\
\hline 12 & Caligiuri, Paula M. & 4 & 1.83 \\
\hline 12 & Money, R. Bruce & 3 & 1.83 \\
\hline 12 & Buck, Trevor & 4 & 1.83 \\
\hline 15 & Asakawa, Kazuhiro & 3 & 1.50 \\
\hline 15 & Chen, Homin & 2 & 1.50 \\
\hline 15 & Lehrer, Mark & 3 & 1.50 \\
\hline 15 & Peterson, Richard B. & 2 & 1.50 \\
\hline 15 & Punnett, Betty Jane & 2 & 1.50 \\
\hline 15 & Ramamurti, Ravi & 2 & 1.50 \\
\hline 15 & Luthans, Fred & 4 & 1.50 \\
\hline 15 & Stroh, Linda K. & 4 & 1.50 \\
\hline 23 & Beamish, Paul W. & 3 & 1.33 \\
\hline 23 & Brewster, Chris & 3 & 1.33 \\
\hline 23 & Kedia, Ben & 3 & 1.33 \\
\hline 23 & Tung, Rosalie L. & 2 & 1.33 \\
\hline 23 & Tuselmann, Heinz-josef & 2 & 1.33 \\
\hline 23 & Lazarova, Mile & 3 & 1.33 \\
\hline 29 & Novicevic, Milorad M. & 3 & 1.25 \\
\hline 30 & Tihanyi, Laszlo & 3 & 1.20 \\
\hline 31 & Law, Kenneth & 3 & 1.08 \\
\hline 31 & Wong, Chi-Sum & 3 & 1.08 \\
\hline 33 & Filatotchev, Igor & 4 & 1.03 \\
\hline
\end{tabular}

Listing only includes authors with an adjusted count greater than 1.00. Overall, 491 different authors appeared in Journal of World Business over the 11-year time period (average 1.25 appearances, average 0.59 adjusted appearances). Of the 491 authors, 415 authors (84.5\%) appeared only once, 46 authors $(9.4 \%)$ appeared twice and 30 authors $(6.1 \%)$ appeared three times or more.

journals (115 authors who have adjusted appearances greater than or equal to 2.50). As indicated in Table 9, the institutions currently hosting the most prolific faculty are Michigan State University (66 author appearances), followed by Leeds University (50 appearances), Rutgers University (33 appearances), Temple University (29 appearances), and University of Reading (29 appearances).

Among Asia Pacific schools, Chinese University of Hong Kong hosts the most prolific faculty (26 appearances), followed by Melbourne Business School (24 appearances). Hong Kong Polytechnic University, National University of Singapore, and the University of Hong Kong are all ranked among the top 20. These Asia Pacific institutions play a critical role on advancement of international business research and deserve a special attention for future years. 
Table 5 Most prolific authors in International Marketing Review (1996-2006): by adjusted count.

\begin{tabular}{|c|c|c|c|}
\hline Rank & Author & Count & Adjusted \\
\hline 1 & Malhotra, Naresh K. & 7 & 2.70 \\
\hline 2 & Sivakumar, K. & 4 & 2.50 \\
\hline 2 & Samiee, Saeed & 5 & 2.50 \\
\hline 2 & Diamantopoulos, Adamantios & 4 & 2.20 \\
\hline 5 & Bennett, Roger & 2 & 2.00 \\
\hline 5 & Holden, Nigel & 2 & 2.00 \\
\hline 5 & Nakata, Cheryl & 3 & 2.00 \\
\hline 5 & Yeoh, Poh-Lin & 2 & 2.00 \\
\hline 5 & Ford, John B. & 4 & 2.00 \\
\hline 10 & Clarke, Irvine III & 3 & 1.83 \\
\hline 10 & Quinn, Barry & 3 & 1.83 \\
\hline 10 & Griffith, David A. & 5 & 1.83 \\
\hline 13 & Leonidou, Leonidas C. & 3 & 1.75 \\
\hline 14 & Schlegelmilch, Bodo B. & 4 & 1.67 \\
\hline 14 & Balabanis, George & 3 & 1.67 \\
\hline 14 & Rawwas, Mohammed Y. A. & 3 & 1.67 \\
\hline 17 & Javalgi, Rajshekhar & 4 & 1.58 \\
\hline 18 & Albers-Miller, Nancy D. & 2 & 1.50 \\
\hline 18 & Douglas, Susan P. & 3 & 1.50 \\
\hline 18 & Jaffe, Eugene D. & 2 & 1.50 \\
\hline 18 & Jeong, Insik & 2 & 1.50 \\
\hline 18 & Katsikeas, Constantine S. & 2 & 1.50 \\
\hline 18 & Murray, Janet Y. & 2 & 1.50 \\
\hline 18 & Okoroafo, Sam C. & 2 & 1.50 \\
\hline 18 & Paliwoda, Stanley J. & 2 & 1.50 \\
\hline 18 & Shaw, Vivienne & 2 & 1.50 \\
\hline 18 & Walters, Peter G. P. & 2 & 1.50 \\
\hline 18 & Wright, Len Tiu & 2 & 1.50 \\
\hline 29 & Singhapakdi, Anusorn & 4 & 1.42 \\
\hline 30 & Agarwal, James & 4 & 1.37 \\
\hline 31 & Calantone, Roger J. & 3 & 1.33 \\
\hline 31 & Knight, Gary A. & 3 & 1.33 \\
\hline 31 & Purchase, Sharon & 2 & 1.33 \\
\hline 31 & Robson, Matthew J. & 3 & 1.33 \\
\hline 31 & Shankarmahesh, Mahesh N. & 2 & 1.33 \\
\hline 31 & Whitelock, Jeryl & 3 & 1.33 \\
\hline 31 & Wood, Van R. & 3 & 1.33 \\
\hline 38 & McNaughton, Rod B. & 2 & 1.30 \\
\hline 39 & Souchon, Anne L. & 3 & 1.20 \\
\hline 40 & Peterson, Mark & 3 & 1.17 \\
\hline 40 & White, D. Steven & 3 & 1.17 \\
\hline 40 & Zou, Shaoming & 3 & 1.17 \\
\hline 43 & Singh, Nitish & 3 & 1.08 \\
\hline 43 & Taylor, Charles R. & 3 & 1.08 \\
\hline 45 & Axinn, Catherine & 3 & 1.03 \\
\hline
\end{tabular}

Listing only includes authors with an adjusted count greater than 1.00. Overall, 552 different authors appeared in International Marketing Review over the 11-year time period (average 1.28 appearances, average 0.59 adjusted appearances). Of the 552 authors, 439 authors (79.5\%) appeared only once, 82 authors $(14.8 \%)$ appeared twice and 31 authors $(5.6 \%)$ appeared three times or more.

Geographic distribution of most prolific authors and institutions is presented in Table 10. An examination of Table 10 indicates that Asia Pacific institutions are in fact one of the major players of advancement of international business research already. 
Table 6 Most prolific authors in Journal of International Marketing (1996-2006): by adjusted count.

\begin{tabular}{llll}
\hline Rank & Author & Count & Adjusted \\
\hline 1 & Cavusgil, S. Tamer & 10 & 4.44 \\
2 & Solberg, Carl Arthur & 4 & 3.33 \\
3 & Craig, C. Samuel & 7 & 3.00 \\
4 & Shoham, Aviv & 4 & 2.75 \\
4 & Griffith, David A. & 8 & 2.75 \\
6 & Douglas, Susan P. & 6 & 2.67 \\
7 & Czinkota, Michael R. & 3 & 2.50 \\
8 & Dow, Douglas & 2 & 2.00 \\
8 & Hsieh, Ming-Huei & 2 & 2.00 \\
10 & Zou, Shaoming & 5 & 1.87 \\
11 & Murray, Janet Y. & 3 & 1.83 \\
11 & Stottinger, Barbara & 3 & 1.83 \\
11 & Styles, Chris & 3 & 1.83 \\
14 & Katsikeas, Constantine S. & 5 & 1.75 \\
15 & Calantone, Roger J. & 4 & 1.58 \\
16 & Eckhardt, Giana & 2 & 1.50 \\
16 & Jones, Marian V. & 2 & 1.50 \\
16 & Ozsomer, Aysegul & 3 & 1.50 \\
19 & Harvey, Michael G. & 3 & 1.33 \\
20 & Wilkinson, Ian F. & 3 & 1.17 \\
20 & Yaprak, Attila & 3 & 1.17 \\
22 & Myers, Matthew B. & 3 & 1.08 \\
22 & Taylor, Charles R. & 3 & 1.08 \\
24 & Vida, Irena & 3 & 1.03 \\
\hline
\end{tabular}

Listing only includes authors with an adjusted count greater than 1.00. Overall, 387 different authors appeared in Journal of International Marketing over the 11-year time period (average 1.31 appearances, average 0.57 adjusted appearances). Of the 387 authors, 318 authors (82.1\%) appeared only once, 46 authors $(11.9 \%)$ appeared twice and 23 authors $(6.0 \%)$ appeared three times or more.

\section{Discussion}

Most prolific authors

With regard to the first research question, the results in Table 8 identify those authors who have been the most frequent contributors to international business research over the 11-year period. These 114 authors represent the top $4.57 \%$ of all authors publishing in the leading six international business journals over this time period. The magnitude of contribution of these leading authors (all achieving a 2.50 adjusted appearance or greater) can best be viewed in comparison to the average adjusted appearance of 0.80 . Further of note, given that these journals have different audiences and objectives, Table 8 can be viewed as recognizing those scholars who publish across a broad spectrum of international business journals. In addition, given the differences across the journals, it is important to consider individual author contributions by journal. Tables 2, 3, 4, 5, 6 and 7 also provide unique insights of author contribution to specific journals and fields and as such provide a more detailed view of individual contributions in light of journal differences. 
Table 7 Most prolific authors in International Business Review (1996-2006): by adjusted count.

\begin{tabular}{|c|c|c|c|}
\hline Author & & Count & Adjusted \\
\hline 1 & Buckley, Peter J. & 8 & 4.67 \\
\hline 2 & Dunning, John H. & 5 & 4.00 \\
\hline 3 & Tang, Gordon Y.N. & 5 & 3.50 \\
\hline 4 & Vachani, Sushil & 3 & 3.00 \\
\hline 4 & Forsgren, Mats & 6 & 3.00 \\
\hline 6 & Glaister, Keith W. & 6 & 2.83 \\
\hline 7 & Chetty, Sylvie & 5 & 2.50 \\
\hline 7 & Cavusgil, S. Tamer & 8 & 2.50 \\
\hline 9 & Brock, David M. & 4 & 2.33 \\
\hline 9 & Fletcher, Richard & 3 & 2.33 \\
\hline 11 & Ellis, Paul & 2 & 2.00 \\
\hline 11 & Eriksson, Kent & 4 & 2.00 \\
\hline 11 & Madhok, Anoop & 2 & 2.00 \\
\hline 11 & Mudambi, Ram & 3 & 2.00 \\
\hline 11 & Riahi-Belkaoui, Ahmed & 2 & 2.00 \\
\hline 11 & Taggart, James H. & 2 & 2.00 \\
\hline 11 & Tsang, Eric W. K. & 2 & 2.00 \\
\hline 18 & Welch, Lawrence S. & 5 & 1.92 \\
\hline 19 & Fey, Carl F. & 3 & 1.83 \\
\hline 19 & Jain, Subhash C. & 3 & 1.83 \\
\hline 19 & Papanastassiou, Marina & 3 & 1.83 \\
\hline 19 & Pearce, Robert & 3 & 1.83 \\
\hline 23 & Andersson, Ulf & 4 & 1.67 \\
\hline 24 & Brouthers, Lance Eliot & 4 & 1.58 \\
\hline 25 & Ang, Swee Hoon & 2 & 1.50 \\
\hline 25 & Budhwar, Pawan S. & 2 & 1.50 \\
\hline 25 & Clegg, Jeremy & 2 & 1.50 \\
\hline 25 & Hadjikhani, Amjad & 2 & 1.50 \\
\hline 25 & Harris, Simon & 2 & 1.50 \\
\hline 25 & Harzing, Anne-Wil & 2 & 1.50 \\
\hline 25 & Leonidou, Leonidas C. & 2 & 1.50 \\
\hline 25 & McNaughton, Rod B. & 2 & 1.50 \\
\hline 25 & Pla-Barber, Jose & 2 & 1.50 \\
\hline 25 & Shum, Wai Cheong & 3 & 1.50 \\
\hline 25 & Teegen, Hildy & 2 & 1.50 \\
\hline 25 & Ulgado, Francis M. & 2 & 1.50 \\
\hline 25 & Pedersen, Torben & 4 & 1.50 \\
\hline 38 & Welch, Denice E. & 4 & 1.42 \\
\hline 39 & Manrai, Ajay K. & 4 & 1.41 \\
\hline 39 & Manrai, Lalita A. & 4 & 1.41 \\
\hline 41 & Ebrahimi, Bahman P. & 2 & 1.33 \\
\hline 41 & Lau, Chung-Ming & 3 & 1.33 \\
\hline 41 & Mayrhofer, Ulrike & 2 & 1.33 \\
\hline 41 & Selmer, Jan & 2 & 1.33 \\
\hline 45 & Qian, Gongming & 2 & 1.25 \\
\hline 45 & Strange, Roger & 3 & 1.25 \\
\hline 45 & Welch, Catherine & 2 & 1.25 \\
\hline 48 & Cheng, Louis T. W. & 3 & 1.17 \\
\hline 48 & Newburry, William & 3 & 1.17 \\
\hline 50 & Birkinshaw, Julian & 3 & 1.08 \\
\hline 50 & Brouthers, Keith D. & 3 & 1.08 \\
\hline
\end{tabular}

Listing only includes authors with an adjusted count greater than 1.00. Overall, 616 different authors appeared in International Business Review over the 11-year time period (average 1.29 appearances, average 0.62 adjusted appearances). Of the 616 authors, 504 authors $(81.8 \%$ ) appeared only once, 77 authors $(12.5 \%)$ appeared twice and 35 authors (5.7\%) appeared three times or more. 
Table 8 Most prolific authors in the six leading international business journals (1996-2006): by adjusted count.

\begin{tabular}{|c|c|c|c|c|}
\hline Rank & Author & Adjusted & Count & Current institution \\
\hline 1 & Luo, Yadong & 16.75 & 23 & University of Miami, USA \\
\hline 2 & Buckley, Peter J. & 15.25 & 27 & Leeds University, UK \\
\hline 3 & Cavusgil, S. Tamer & 12.03 & 29 & Michigan State University, USA \\
\hline 4 & Griffith, David A. & 9.17 & 23 & Michigan State University, USA \\
\hline 5 & Dunning, John H. & 8.00 & 9 & University of Reading/Rutgers University, UK \\
\hline 6 & Harvey, Michael G. & 7.75 & 15 & University of Mississippi, USA \\
\hline 7 & Katsikeas, Constantine S. & 6.92 & 15 & Leeds University, UK \\
\hline 8 & Tsang, Eric W. K. & 6.67 & 8 & University of Texas at Dallas, USA \\
\hline 9 & Pan, Yigang & 6.33 & 13 & York University, Canada \\
\hline 10 & Leonidou, Leonidas C. & 6.25 & 11 & University of Cyprus, Cyprus \\
\hline 11 & Brouthers, Lance Eliot & 6.08 & 13 & University of Texas at El Paso, USA \\
\hline 11 & Mudambi, Ram & 6.08 & 11 & Temple University, USA \\
\hline 13 & Rugman, Alan M. & 5.83 & 11 & Indiana University, USA \\
\hline 14 & Czinkota, Michael R. & 5.50 & 8 & Georgetown University, USA \\
\hline 14 & Ellis, Paul & 5.50 & 7 & Hong Kong Polytechnic University, Hong Kong \\
\hline 16 & Meyer, Klaus & 5.33 & 8 & University of Reading, UK \\
\hline 17 & Douglas, Susan P. & 5.17 & 11 & New York University, USA \\
\hline 17 & Kotabe, Masaaki & 5.17 & 13 & Temple University, USA \\
\hline 17 & Peng, Mike W. & 5.17 & 9 & University of Texas at Dallas, USA \\
\hline 20 & Beamish, Paul W. & 5.08 & 12 & University of Western Ontario, Canada \\
\hline 20 & Birkinshaw, Julian & 5.08 & 11 & London Business School, UK \\
\hline 22 & Casson, Mark & 5.00 & 7 & University of Reading, UK \\
\hline 22 & Craig, C. Samuel & 5.00 & 11 & New York University, USA \\
\hline 24 & $\begin{array}{c}\text { Diamantopoulos, } \\
\text { Adamantios }\end{array}$ & 4.59 & 11 & Universität Wien, Austria \\
\hline 25 & Tse, David & 4.58 & 12 & The University of Hong Kong, Hong Kong \\
\hline 26 & Knight, Gary A. & 4.50 & 9 & Florida State University, USA \\
\hline 26 & Murray, Janet Y. & 4.50 & 8 & University of Missouri - St. Louis, USA \\
\hline 28 & Shenkar, Oded & 4.42 & 8 & The Ohio State University, USA \\
\hline 28 & Welch, Lawrence S. & 4.42 & 11 & Melbourne Business School, Australia \\
\hline 30 & Glaister, Keith W. & 4.33 & 9 & University of Sheffield, UK \\
\hline 30 & Newburry, William & 4.33 & 9 & Rutgers University, USA \\
\hline 30 & Samiee, Saeed & 4.33 & 9 & University of Tulsa, USA \\
\hline 30 & Selmer, Jan & 4.33 & 5 & Aarhus School of Business, Denmark \\
\hline 30 & Solberg, Carl Arthur & 4.33 & 6 & Norwegian School of Management, Norway \\
\hline 35 & Brouthers, Keith D. & 4.25 & 9 & King's College London, UK \\
\hline 36 & Verbeke, Alain & 4.17 & 9 & University of Calgary, Canada \\
\hline 37 & Harzing, Anne-Wil & 4.00 & 5 & University of Melbourne, Australia \\
\hline 37 & Merchant, Hemant & 4.00 & 4 & Simon Frasier University, Canada \\
\hline 37 & Nachum, Lilach & 4.00 & 5 & Baruch College - CUNY, USA \\
\hline 37 & Taggart, James H. & 4.00 & 4 & University of Glasgow, UK \\
\hline 41 & Welch, Denice E. & 3.92 & 9 & Melbourne Business School, Australia \\
\hline 42 & Fey, Carl F. & 3.87 & 8 & Stockholm School of Economics, Sweden \\
\hline 42 & Pedersen, Torben & 3.87 & 10 & Copenhagen Business School, Denmark \\
\hline 44 & Delios, Andrew & 3.83 & 8 & National University of Singapore, Singapore \\
\hline 44 & Stottinger, Barbara & 3.83 & 7 & Wirtschaftsuniversität Wien, Austria \\
\hline 46 & Shoham, Aviv & 3.75 & 7 & University of Haifa, Israel \\
\hline 47 & Buck, Trevor & 3.66 & 9 & Loughborough University, UK \\
\hline 48 & Myers, Matthew B. & 3.58 & 6 & University of Tennessee, USA \\
\hline 49 & Ang, Swee Hoon & 3.50 & 4 & National University of Singapore, Singapore \\
\hline 49 & Dow, Douglas & 3.50 & 4 & Melbourne Business School, Australia \\
\hline 49 & Forsgren, Mats & 3.50 & 7 & Uppsala University, Sweden \\
\hline 49 & Grosse, Robert & 3.50 & 5 & Thunderbird, USA \\
\hline 49 & Parkhe, Arvind & 3.50 & 5 & Temple University, USA \\
\hline
\end{tabular}


Table 8 (continued)

\begin{tabular}{|c|c|c|c|c|}
\hline Rank & Author & Adjusted & Count & Current institution \\
\hline 49 & Ramamurti, Ravi & 3.50 & 4 & Northeastern University, USA \\
\hline 49 & Sivakumar, K. & 3.50 & 6 & Lehigh University, USA \\
\hline 49 & Tang, Gordon Y.N. & 3.50 & 5 & Hong Kong Baptist University, Hong Kong \\
\hline 57 & Balabanis, George & 3.42 & 6 & City University, London, UK \\
\hline 57 & Clegg, Jeremy & 3.42 & 8 & Leeds University, UK \\
\hline 59 & Malhotra, Naresh K. & 3.37 & 9 & Georgia Institute of Technology, USA \\
\hline 59 & Zou, Shaoming & 3.37 & 9 & University of Missouri at Columbia, USA \\
\hline 61 & Chetty, Sylvie & 3.33 & 7 & Massey University, New Zealand \\
\hline 61 & Coviello, Nicole $\mathbf{E}$. & 3.33 & 6 & University of Auckland, New Zealand \\
\hline 61 & Lenartowicz, Tomasz & 3.33 & 7 & Florida Atlantic University, USA \\
\hline 61 & Makino, Shige & 3.33 & 8 & Chinese University of Hong Kong, Hong Kong \\
\hline 61 & Money, R. Bruce & 3.33 & 5 & Brigham Young University, USA \\
\hline 61 & Schlegelmilch, Bodo B. & 3.33 & 8 & Wirtschaftsuniversität Wien, Austria \\
\hline 61 & Vachani, Sushil & 3.33 & 4 & Boston University, USA \\
\hline 61 & Zhao, Hongxin & 3.33 & 8 & Saint Louis University, USA \\
\hline 69 & McNaughton, Rod B. & 3.30 & 5 & University of Waterloo, Canada \\
\hline 70 & Eriksson, Kent & 3.25 & 8 & KTH-The Royal Institute of Technology, Sweden \\
\hline 70 & Lin, Xiaohua & 3.25 & 6 & University of Windsor, Canada \\
\hline 72 & Brewster, Chris & 3.17 & 6 & Henley Management College, UK \\
\hline 72 & Madhok, Anoop & 3.17 & 5 & University of Utah, USA \\
\hline 72 & Puffer, Sheila M. & 3.17 & 6 & Northeastern University, USA \\
\hline 75 & Johanson, Jan & 3.08 & 8 & Uppsala University, Sweden \\
\hline 75 & Qian, Gongming & 3.08 & 6 & Chinese University of Hong Kong, Hong Kong \\
\hline 77 & Johnson, James P. & 3.03 & 8 & Rollins College, USA \\
\hline 77 & Ulgado, Francis M. & 3.03 & 5 & Georgia Tech, USA \\
\hline 79 & Andersen, Poul Houman & 3.00 & 4 & Aarhus School of Business, Denmark \\
\hline 79 & Hadjikhani, Amjad & 3.00 & 4 & Uppsala University, Sweden \\
\hline 79 & Meschi, Pierre-Xavier & 3.00 & 4 & Université de la Méditerranée, France \\
\hline 79 & Pla-Barber, Jose & 3.00 & 4 & University of Valencia, Spain \\
\hline 79 & Walters, Peter G. P. & 3.00 & 5 & Hong Kong Polytechnic University, Hong Kong \\
\hline 79 & Yeoh, Poh-Lin & 3.00 & 3 & Bentley College, USA \\
\hline 85 & Calantone, Roger J. & 2.92 & 7 & Michigan State University, USA \\
\hline 85 & Ford, John B. & 2.92 & 7 & Old Dominion University, USA \\
\hline 87 & Bjorkman, Ingmar & 2.87 & 7 & Swedish School of Economics, Sweden \\
\hline 88 & Brock, David M. & 2.83 & 5 & Ben-Gurion University, Israel \\
\hline 88 & Budhwar, Pawan S. & 2.83 & 5 & Aston Business School, UK \\
\hline 88 & Caligiuri, Paula M. & 2.83 & 5 & Rutgers University, USA \\
\hline 88 & Jain, Subhash C. & 2.83 & 4 & University of Connecticut, USA \\
\hline 88 & Lau, Chung-Ming & 2.83 & 7 & Chinese University of Hong Kong, Hong Kong \\
\hline 88 & Mascarenhas, Briance & 2.83 & 4 & Rutgers University, USA \\
\hline 88 & Pearce, Robert & 2.83 & 5 & University of Reading, UK \\
\hline 88 & Reuer, Jeffrey J. & 2.83 & 4 & University of North Carolina, USA \\
\hline 88 & Roth, Kendall & 2.83 & 6 & University of South Carolina, USA \\
\hline 88 & Sambharya, Rakesh B. & 2.83 & 6 & Rutgers University - Camden, USA \\
\hline 88 & Styles, Chris & 2.83 & 5 & University of Sydney, Australia \\
\hline 99 & Husted, Bryan W. & 2.75 & 4 & Instituto De Empresa, Spain \\
\hline 99 & Nakata, Cheryl & 2.75 & 5 & University of Illinois - Chicago, USA \\
\hline 101 & Au, Kevin & 2.67 & 5 & Chinese University of Hong Kong, Hong Kong \\
\hline 101 & Contractor, Farok & 2.67 & 6 & Rutgers University, USA \\
\hline 101 & Inkpen, Andrew C. & 2.67 & 5 & Thunderbird, USA \\
\hline 101 & Kundu, Sumit K. & 2.67 & 6 & Florida International University, USA \\
\hline 101 & Kwok, Chuck C. & 2.67 & 6 & University of South Carolina, USA \\
\hline 106 & Teegen, Hildy & 2.58 & 6 & George Washington University, USA \\
\hline 107 & Andersen, Otto & 2.50 & 4 & Agder University College, Norway \\
\hline 107 & Andersson, Ulf & 2.50 & 6 & Uppsala University, Sweden \\
\hline
\end{tabular}


Table 8 (continued)

\begin{tabular}{|c|c|c|c|c|}
\hline Rank & Author & Adjusted & Count & Current institution \\
\hline 107 & Aulakh, Preet S. & 2.50 & 7 & York University, Canada \\
\hline 107 & Cho, Kang Rae & 2.50 & 5 & University of Colorado at Denver, USA \\
\hline 107 & Hult, G. Tomas M. & 2.50 & 7 & Michigan State University, USA \\
\hline 107 & Nigh, Douglas ${ }^{\mathrm{a}}$ & 2.50 & 5 & University of South Carolina, USA \\
\hline 107 & Petersen, Bent & 2.50 & 6 & Copenhagen Business School, Denmark \\
\hline 107 & Sullivan, Daniel P. & 2.50 & 3 & University of Delaware, USA \\
\hline
\end{tabular}

Adjusted count equal to or greater than 2.50. For APJM purposes, bold typeface is used to identify authors affiliated with Asia Pacific schools. The remaining 2,381 authors had an adjusted appearance score of between 0.01 and 2.48 .

${ }^{\mathrm{a}}$ Deceased

\section{Leading institutions}

In terms of leading institutions, we believe that it is important to look both at the output of authors at each institution and the number of prolific authors (authors who have adjusted appearances equal to or greater than 2.50). An examination of Table 9 shows that institutions hosting the most prolific authors (based upon number of appearances) in terms of output are Michigan State University, Leeds University, Rutgers University, Temple University, and the University of Reading. Furthermore, an examination of Table 9 in terms of the number of scholars currently hosted at the aforementioned institutions implies a specific focus of these institutions on international business. Given the number of prolific authors at these institutions, it can be argued that these institutions may hold considerable influence on the direction of international business research. If such distinctive leadership has resulted from a deliberate or programmatic effort, these institutions should receive special credit for facilitating advancement of international business knowledge.

An examination of Table 9 also indicates that Asia Pacific institutions hosting the most prolific authors ranked high on the list, having two institutions in the top 10 and five institutions in the top 20. This finding suggests that Asia Pacific institutions' importance in driving the international business research agenda is quite considerable. Given the fact that few institutions maintain IB departments (Griffith et al., 2008), Asia Pacific institutions' success in the ranking may imply a specific focus on international business research.

While the current state of author affiliation and author productivity provide some insights into which institutions and individuals may influence future scholarly work in international business, it is important to note two caveats. First, past productivity is no indication of future productivity and, therefore, it is unclear whether those listed as prolific authors today will continue to contribute to the field at the same rate. It is not unusual to see productivity fall off when researchers leave academia to join industry or pursue an administrative career. Second, mobility in academia appears to be increasing and therefore it is often difficult for institutions to retain top scholars from being recruited by other institutions wishing to develop (or reestablish) a leadership position. As such, increased author mobility places increased 
Table 9 Institutions currently hosting the most prolific authors in the leading international business journals (1996-2006).

\begin{tabular}{|c|c|c|c|}
\hline Rank & Institution & Prolific authors & Coun \\
\hline 1 & Michigan State University, USA & 4 & 66 \\
\hline 2 & Leeds University, UK & 3 & 50 \\
\hline 3 & Rutgers University ${ }^{\mathrm{a}}$, USA & 5 & 33 \\
\hline 4 & Temple University, USA & 3 & 29 \\
\hline 4 & University of Reading ${ }^{\mathrm{a}}$, UK & 4 & 29 \\
\hline 6 & Chinese University of Hong Kong, HK & 4 & 26 \\
\hline 7 & Uppsala University, Sweden & 4 & 25 \\
\hline 8 & Melbourne Business School, Australia & 3 & 24 \\
\hline 9 & University of Miami, USA & 1 & 23 \\
\hline 10 & New York University, USA & 2 & 22 \\
\hline 11 & York University, Canada & 2 & 20 \\
\hline 12 & University of Texas at Dallas, USA & 2 & 17 \\
\hline 13 & Copenhagen Business School, Denmark & 2 & 16 \\
\hline 14 & University of Mississippi, USA & 1 & 15 \\
\hline 14 & Wirtschaftsuniversität Wien, Austria & 2 & 15 \\
\hline 16 & University of Texas at El Paso, USA & 1 & 13 \\
\hline 17 & Hong Kong Polytechnic University, HK & 2 & 12 \\
\hline 17 & National University of Singapore, Singapore & 2 & 12 \\
\hline 17 & Old Dominion University, USA & 2 & 12 \\
\hline 17 & The University of Hong Kong, HK & 1 & 12 \\
\hline 17 & University of South Carolina, USA & 2 & 12 \\
\hline 17 & University of Western Ontario, Canada & 1 & 12 \\
\hline 23 & Indiana University, USA & 1 & 11 \\
\hline 23 & London Business School, UK & 1 & 11 \\
\hline 23 & Universität Wien, Austria & 1 & 11 \\
\hline 23 & University of Cyprus, Cyprus & 1 & 11 \\
\hline 27 & Northeastern University, USA & 2 & 10 \\
\hline 27 & Thunderbird, USA & 2 & 10 \\
\hline 29 & Aarhus School of Business, Denmark & 2 & 9 \\
\hline 29 & Florida State University, USA & 1 & 9 \\
\hline 29 & Georgia Institute of Technology, USA & 1 & 9 \\
\hline 29 & King's College London, UK & 1 & 9 \\
\hline 29 & Loughborough University, UK & 1 & 9 \\
\hline 29 & University of Calgary, Canada & 1 & 9 \\
\hline 29 & University of Missouri at Columbia, USA & 1 & 9 \\
\hline 29 & University of Sheffield, UK & 1 & 9 \\
\hline 29 & University of Tulsa, USA & 1 & 9 \\
\hline 38 & Georgetown University, USA & 1 & 8 \\
\hline 38 & KTH-The Royal Institute of Technology, Sweden & 1 & 8 \\
\hline 38 & Rollins College, USA & 1 & 8 \\
\hline 38 & Saint Louis University, USA & 1 & 8 \\
\hline 38 & Stockholm School of Economics, Sweden & 1 & 8 \\
\hline 38 & The Ohio State University, USA & 1 & 8 \\
\hline 38 & University of Missouri - St. Louis, USA & 1 & 8 \\
\hline 45 & Florida Atlantic University, USA & 1 & 7 \\
\hline 45 & Massey University, New Zealand & 1 & 7 \\
\hline 45 & Swedish School of Economics, Sweden & 1 & 7 \\
\hline 45 & University of Haifa, Israel & 1 & 7 \\
\hline 49 & City University, London, UK & 1 & 6 \\
\hline 49 & Florida International University, USA & 1 & 6 \\
\hline 49 & George Washington University, USA & 1 & 6 \\
\hline 49 & Henley Management College, UK & 1 & 6 \\
\hline 49 & Lehigh University, USA & 1 & 6 \\
\hline 49 & Norwegian School of Management, Norway & 1 & 6 \\
\hline 49 & Rutgers University - Camden, USA & 1 & 6 \\
\hline 49 & University of Auckland, New Zealand & 1 & 6 \\
\hline
\end{tabular}


Table 9 (continued)

\begin{tabular}{llll}
\hline Rank & Institution & Prolific authors & Count \\
\hline 49 & University of Tennessee, USA & 1 & 6 \\
49 & University of Windsor, Canada & 1 & 6 \\
59 & Aston Business School, UK & 1 & 5 \\
59 & Baruch College - CUNY, USA & 1 & 5 \\
59 & Ben-Gurion University, Israel & 1 & 5 \\
59 & Brigham Young University, USA & 1 & 5 \\
59 & Georgia Tech, USA & 1 & 5 \\
$\mathbf{5 9}$ & Hong Kong Baptist University, HK & 1 & 5 \\
59 & University of Colorado at Denver, USA & 1 & 5 \\
59 & University of Illinois - Chicago, USA & 1 & 5 \\
$\mathbf{5 9}$ & University of Melbourne, Australia & 1 & 5 \\
$\mathbf{5 9}$ & University of Sydney, Australia & 1 & 4 \\
59 & University of Utah, USA & 1 & 4 \\
59 & University of Waterloo, Canada & 1 & 4 \\
71 & Agder University College, Norway & 1 & 4 \\
71 & Bentley College, USA & 1 & 4 \\
71 & Boston University, USA & 1 & 4 \\
71 & Instituto de Empresa, Spain & 1 & 4 \\
71 & Simon Frasier University, Canada & 1 & 4 \\
71 & Université de la Méditerranée, France & 1 & 4 \\
71 & University of Connecticut, USA & 1 & 4 \\
71 & University of Glasgow, UK & 1 & 1 \\
71 & University of North Carolina, USA & 1 & 5 \\
81 & University of Valencia, Spain & 1 & 5 \\
\hline
\end{tabular}

For APJM purposes, bold typeface is used to identify Asia Pacific schools

${ }^{a}$ John Dunning holds dual appointments (both schools were credited with affiliation and appearances)

Table 10 Geographic distribution of institutions currently hosting the most prolific authors in the six leading international business journals (1996-2006).

\begin{tabular}{lllll}
\hline Rank & Country & Number of institutions & Prolific authors & Count \\
\hline 1 & United States & 38 & 26 & 431 \\
2 & United Kingdom & 10 & 15 & 138 \\
3 & Canada & 6 & 7 & 56 \\
4 & Hong Kong & 4 & 8 & 55 \\
5 & Sweden & 4 & 7 & 48 \\
6 & Australia & 3 & 5 & 34 \\
7 & Austria & 2 & 3 & 26 \\
8 & Denmark & 2 & 4 & 25 \\
9 & New Zealand & 2 & 2 & 13 \\
10 & Israel & 2 & 2 & 12 \\
11 & Singapore & 1 & 2 & 12 \\
12 & Cyprus & 1 & 1 & 11 \\
13 & Norway & 2 & 2 & 8 \\
14 & Spain & 2 & 2 & 4 \\
15 & France & 1 & 1 & 487 \\
Subtotal: & North America & 44 & & 270 \\
Region & Europe & 24 & 33 & 114 \\
& Asia & 10 & 35 & \\
& & & 17 & \\
\hline
\end{tabular}


emphasis on identifying the specific individual scholars who contribute to the field as opposed to the institutions they represent.

\section{Limitations and follow-up studies}

Although the present study provides important insights into the state of scholarship in international business, it is nevertheless key to recognize some limitations to this work. First, although every effort was made to present an objective assessment of authorship or research articles, subjective decisions were made with respect to the inclusion or exclusion of certain types of articles. For example, editorials and guest editorials were not included in the analysis due to their lack of peer review. While the exclusion of editorials follows prior practice (e.g., Inkpen \& Beamish, 1994), a number of cross-journal inconsistencies required decisions to be made on various aspects of all journals. The exclusion of certain types of articles should not be inferred as a judgment related to quality of the articles or contribution to the field, but rather a decision related to consistency in review processes for the articles examined.

Second, to determine authorship productivity, the leading international business journals were weighted equally. The utilization of equal journal weighting presents problems due to differences in acceptance rates, journal focus, and so on. Thus, interpretation of the rankings of individual authors should be viewed in detail (Tables 2, 3, 4, 5, 6 and 7) to determine specific contributions within each area. This becomes more important in such areas as international accounting and international finance that, by most measures, are underrepresented in the leading six international business journals. Furthermore, this suggests that accounting and finance scholars may find greater receptivity to their efforts in their mainstream functional journals.

Third, a particular 11-year time period (1996-2006) was selected and selecting another time period may change the findings. The productivity of any single author varies based upon their individual research career cycles, editorial roles which may make them ineligible for publishing in certain journals, and so on. Clearly, another time period could identify a different set of author productivity and institutions.

A plethora of additional limitations can be raised. Research productivity is a very sensitive issue, one that the present authors have taken seriously and have attempted to handle with appropriate diligence and care. However, errors and omissions may be present. For example, as author names were used, any name changes (whether full name change, first or last name change, inclusion or deletion of middle names, etc.) may have resulted in inconsistencies. To minimize any errors or omissions, multiple individuals were consulted to check the data. In addition, not focusing on citations is a limitation. Peng and Zhou (2006) show that the list of most prolific authors does not strongly correlate with the list of most cited authors. Griffith et al. (2008) and Pleggenkuhle et al. (2007) have interesting discussions on citations. Another limitation is that our global approach, focusing on all areas in IB, has prevented us from taking a more detailed look at research focusing on specific geographic regions, such as China (Quer, Claver, \& Rienda, 2007) and Central and Eastern Europe (Meyer \& Peng, 2005). 


\section{Conclusion}

The present study provides a contemporary snapshot of the leading authors and institutions in international business. The findings support the view that the field of international business has now reached prominence in academic circles; an increasing number of business schools now claim leadership in this field and stress it in competitive positioning. The prolific nature of scholarship in the field of international business (e.g., 2,495 different authors publishing in the leading six journals from 1996 to 2006 with articles being cited 8,598 times), combined with the institutional analysis of Kumar and Kundu (2004) and the editorial review board membership study of Chan et al. (2005), indicate that the discipline of international business has arrived in force. The results of the institutional analysis suggest that the academic recognition of the field of international business is worldwide. Thus, it appears that international business has so far produced a critical mass of scholars with diverse institutional perspectives to address the burning questions of the field in years to come.

Finally, the findings have important implications for business education. International business has not only become a prominent discipline among academics worldwide, but a recognized area of expertise among executives as well. As scholarship in the discipline is a key driver of institutional reputation (along with curriculum), institutions wishing to gain prominence in this area could do so by assembling a critical mass of prolific international business scholars. Certain institutions have already made progress in this direction. Interestingly, as even those institutions that rank among the most prominent in terms of international business research productivity and the number of scholars hosted, no institution currently hosts more than five of the 115 most prolific authors. As such, opportunities exist for other institutions seeking prominence in the area by assembling a critical mass of active researchers. By doing so, an institution may not only be able to enhance its overall reputation, but also create an opportunity for its faculty to have an impact on the development of international business as a scholarly discipline.

\section{References}

Chan, K.C., Fung, H., \& Lai, P. 2005. Membership of editorial boards and rankings of schools with international business orientation. Journal of International Business Studies, 36: 452-469.

Dubois, F. L., \& Reeb, D. M. 2000. Ranking the international business journals. Journal of International Business Studies, 31: 689-704.

Griffith, D. A., Cavusgil, S. T., \& Xu, S. 2008. Emerging themes in international business research. Journal of International Business Studies, (in press).

Inkpen, A.C., \& Beamish, P. W. 1994. An analysis of twenty-five years of research in the Journal of International Business Studies. Journal of International Business Studies, 25: 703-713.

Kumar, V., \& Kundu, S. K. 2004. Ranking the international business schools: Faculty publication as the measure. Management International Review, 44: 213-228.

Meyer, K. E., \& Peng, M. W. 2005. Probing theoretically into Central and Eastern Europe: Transactions, resources and institutions. Journal of International Business Studies, 25: 600-621.

Peng, M. W. 2001. The resource-based view and international business. Journal of Management, 27: 803829.

Peng, M. W., \& Zhou, J. Q. 2006. Most cited authors and articles in global strategy research. Journal of International Management, 12: 490-508.

Springer 
Pleggenkuhle, E. G., Aroul, R. R., Sun, S. L., \& Su, Y. 2007. The adolescence of Asia management research: APJM, 1997-2006. Asia Pacific Journal of Management, 24: 467-490.

Quer, D., Claver, E., \& Rienda, L. 2007. Business and management in China: A review of empirical research in leading international journals. Asia Pacific Journal of Management, 24: 359-384.

Trieschmann, J. S., Dennis, A. R., Northcraft, G. R., \& Niemi, A. W. 2000. Serving multiple constituencies in business schools: M.B.A. program versus research performance. Academy of Management Journal, 43: 1130-1141.

Shichun Xu ( $\mathrm{PhD}$, Michigan State University) is an assistant professor of marketing at University of Tennessee. His research interests are in the areas of marketing strategy, strategic alliance management, and marketing in emerging economies.

Goksel Yalcinkaya ( $\mathrm{PhD}$, Michigan State University) is an assistant professor of marketing in the Whittemore School of Business and Economics, University of New Hampshire. His research interests are in the areas of innovation diffusions (both domestic and international), new product launch, and international marketing. His work has previously been published in various academic journals including the Journal of International Marketing and International Marketing Review.

Steven H. Seggie ( $\mathrm{PhD}$, Michigan State University) is an assistant professor of marketing at Bilkent University, Ankara, Turkey. His research interests are in the areas of interorganizational relationships, international and domestic marketing channels, supply chain management, institutional economics and scientometrics. His work has previously been published in various academic journals including the Journal of Business Research and Industrial Marketing Management. 